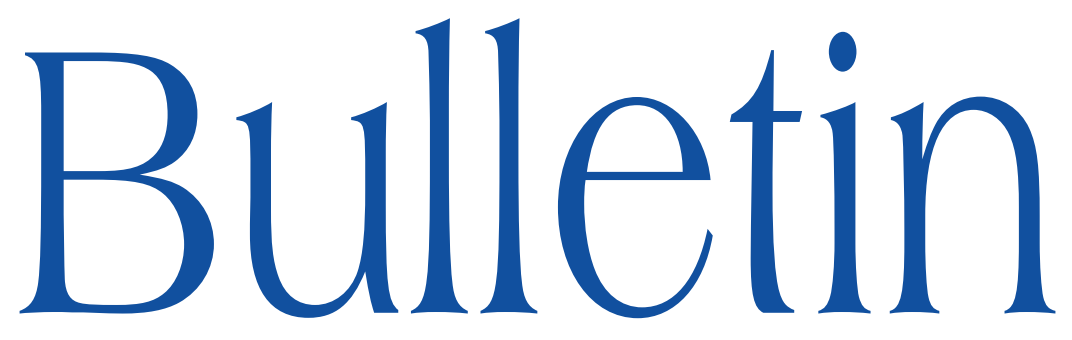

de la SOCIÉTÉ MATHÉMATIQUE DE FRANCE

\title{
COCYCLES OVER A MINIMAL BASE
}

\author{
Artur Avila \& Jairo Bochi
}

\section{Tome 135 Fascicule 3}




\title{
A UNIFORM DICHOTOMY FOR GENERIC SL $(2, \mathbb{R})$ COCYCLES OVER A MINIMAL BASE
}

\author{
By ArTur Avila \& Jairo Bochi
}

\begin{abstract}
We consider continuous $\mathrm{SL}(2, \mathbb{R})$-cocycles over a minimal homeomorphism of a compact set $K$ of finite dimension. We show that the generic cocycle either is uniformly hyperbolic or has uniform subexponential growth.

RÉSUmÉ (Une dichotomie uniforme pour des cocycles à valeurs dans $\mathrm{SL}(2, \mathbb{R})$ audessus d'une dynamique minimale)

On considère des cocycles continus à valeurs dans $\mathrm{SL}(2, \mathbb{R})$ au-dessus d'un homéomorphisme minimal d'un ensemble compact de dimension finie. On montre que le cocycle générique soit est uniformément hyperbolique, soit possède une croissance sousexponentielle uniforme.
\end{abstract}

\section{Introduction}

In this paper we will consider $\mathrm{SL}(2, \mathbb{R})$-valued cocycles over a minimal homeomorphism $f: K \rightarrow K$ of a compact set $K$. Such a cocycle can be defined

Texte recu le 14 novembre 2006 révisé le 23 mars 2007

Artur Avila, CNRS UMR 7599, Laboratoire de Probabilités et Modèles aléatoires, Université Pierre et Marie Curie - Boîte 188, 75252 Paris Cedex 05 (France)

Url : http://www.proba.jussieu.fr/pageperso/artur

- E-mail : artur@math.sunysb.edu

Jairo Bochi, Departamento de Matemática, PUC-Rio, Rio de Janeiro (Brazil)

Url : http://www.mat.puc-rio.br/pessoas/jairo.html

- E-mail : jairo@mat.puc-rio.br

2000 Mathematics Subject Classification. - 37H15.

Key words and phrases. - Cocycle, minimal homeomorphism, uniform hyperbolicity, Lyapunov exponents. 
as a pair $(f, A)$ where $A: K \rightarrow \mathrm{SL}(2, \mathbb{R})$ is continuous. The cocycle acts on $K \times \mathbb{R}^{2}$ by $(x, y) \mapsto(f(x), A(x) \cdot y)$. The iterates of the cocycle are denoted $(f, A)^{n}=\left(f^{n}, A_{n}\right)$.

We say that $(f, A)$ is uniformly hyperbolic if there exists $\varepsilon>0$ and $N>0$ such that $\left\|A_{n}(x)\right\| \geq \mathrm{e}^{\varepsilon n}$ for every $x \in K, n \geq N$. This is equivalent to the existence of a continuous invariant splitting $\mathbb{R}^{2}=E^{u}(x) \oplus E^{s}(x)$ such that vectors in $E^{s}(x)$ are exponentially contracted by forward iteration and vectors in $E^{u}(x)$ are exponentially contracted by backwards iteration - see [7, Prop. 2].

We say that $(f, A)$ has uniform subexponential growth if for every $\varepsilon>0$ there exists $N>0$ such that $\left\|A_{n}(x)\right\| \leq \mathrm{e}^{\varepsilon n}$ for every $x \in K, n \geq N$. This condition is equivalent to the vanishing of the Lyapunov exponent for all $f$-invariant probability measures (see Proposition 1 below). We recall that the Lyapunov exponent of the cocycle $(f, A)$ with respect to an $f$-invariant probability measure $\mu$ is defined as

$$
L(f, A, \mu)=\lim \frac{1}{n} \int_{K} \log \left\|A_{n}\right\| \mathrm{d} \mu .
$$

We say that a compact set $K$ has finite dimension if it is homeomorphic to a subset of some $\mathbb{R}^{n}$. For instance, compact subsets of manifolds (assumed as usual to be Hausdorff and second countable) have finite dimension. (For definitions of dimension and results concerning embedding in $\mathbb{R}^{n}$, see e.g. [6].)

Theorem 1. - Let $f: K \rightarrow K$ be a minimal homeomorphism of a compact set of finite dimension. Then for generic continuous $A: K \rightarrow \mathrm{SL}(2, \mathbb{R})$, either $(f, A)$ is uniformly hyperbolic or $(f, A)$ has uniform subexpoential growth.

In the case where $f$ is a minimal uniquely ergodic homeomorphism, Theorem 1 is contained in [1], which shows that if $f: K \rightarrow K$ is a homeomorphism, then for any ergodic $f$-invariant probability $\mu$, the generic continuous $A: K \rightarrow \mathrm{SL}(2, \mathbb{R})$ is such that either $L(f, A, \mu)=0$ or the restriction of $(f, A)$ to the support of $\mu$ is uniformly hyperbolic. In general a minimal homeomorphism may admit uncountably many ergodic invariant probability measures, and in this paper we show how to treat them all at the same time. In the perturbation arguments, one often allows for loss of control in some sets, and this must be compensated by showing that such sets can be selected small. The notion of smallness needed for our purposes involves simultaneous conditions on all $f$-invariant probability measures.

It is unclear whether the hypothesis that $K$ has finite dimension is actually necessary. The following example shows that the minimality hypothesis cannot be significantly weakened. 
Example 1.1. - Let $S^{3}$ be identified with $\mathbb{C}_{*}^{2} / \mathbb{R}_{+}$(here $\mathbb{C}_{*}^{2}=\mathbb{C}^{2} \backslash\{(0,0)\}$ and $\mathbb{R}_{+}=\{r \in \mathbb{R} ; r>0\}$ ). Fix some $\alpha \in \mathbb{R}$ with $\alpha / 2 \pi$ irrational and let $f: S^{3} \rightarrow S^{3}$ be given by

$$
(z, w) \longmapsto\left(\mathrm{e}^{i \alpha} z, \mathrm{e}^{i \alpha}(z+w)\right) \bmod \mathbb{R}_{+} .
$$

Notice that if $h: S^{3} \rightarrow \mathbb{C P}^{1}$ with $h(z, w)=w / z$ is the usual Hopf fibration, and $g: \mathbb{C P}^{1} \rightarrow \mathbb{C P}^{1}$ is $g(w)=w+1$, then $h \circ f=g \circ h$. So $f$ has a unique minimal set, namely $S=h^{-1}(\infty)$, where $f$ acts as a irrational rotation. Let $A: S^{3} \rightarrow \mathrm{SL}(2, \mathbb{R})$ be any continuous map whose restriction to $S$ is given by

$$
\left(0, r \mathrm{e}^{i \theta}\right) \longmapsto\left(\begin{array}{cc}
\cos (\theta+\alpha) & -\sin (\theta+\alpha) \\
\sin (\theta+\alpha) & \cos (\theta+\alpha)
\end{array}\right)\left(\begin{array}{ll}
2 & 0 \\
0 & \frac{1}{2}
\end{array}\right)\left(\begin{array}{cc}
\cos \theta & \sin \theta \\
-\sin \theta & \cos \theta
\end{array}\right) .
$$

Then $(f|S, A| S)$ is uniformly hyperbolic: the associated splitting is such that $E^{u}$ and $E^{s}$ are orthogonal and $E^{u}\left(0, r \mathrm{e}^{i \theta}\right)$ is generated by $\left(\begin{array}{c}\cos \theta \\ \sin \theta\end{array}\right)$. This splitting is topologically nontrivial, and hence cannot be extended to the whole $S^{3}$. It follows that $(f, A)$ is not uniformly hyperbolic and does not have uniform subexponential growth, and the same properties hold for any small perturbation of $A$.

Using ideas from [2], [5], one can adapt the arguments of this paper to deal with $\operatorname{GL}(d, \mathbb{R})$-valued cocycles. The conclusion is that for a generic continuous $A: K \rightarrow \mathrm{GL}(d, \mathbb{R})$ and for every $f$-invariant probability measure $\mu$, the Oseledets splitting relative to $\mu$ coincides almost everywhere with the finest dominated splitting of $(f, A)$.

Acknowledgements. - This research was partially conducted during the period the first author served as a Clay Research Fellow. The paper was written while the first author was visiting UFRGS partially supported by Procad/CAPES. The second author is partially supported by $\mathrm{CNPq}$ (Brazil).

\section{Uniform subexponential growth}

Here we prove an equivalence stated at the introduction:

Proposition 1. - Let $f: K \rightarrow K$ be homeomorphism of a compact set $K$. and $A: K \rightarrow \mathrm{SL}(2, \mathbb{R})$ be a continuous map. Then the following are equivalent:

(a) $(f, A)$ has uniform subexponential growth: for every $\varepsilon>0$ there exists $N>0$ such that $\left\|A_{n}(x)\right\| \leq \mathrm{e}^{\varepsilon n}$ for every $x \in K, n \geq N$;

(b) for every $\varepsilon>0$ there exists $n>0$ such that $\left\|A_{n}(x)\right\| \leq \mathrm{e}^{\varepsilon n}$ for every $x \in K$

(c) $L(f, A, \mu)=0$ for every $f$-invariant probability $\mu$. 
In the case that $f$ is uniquely ergodic, the proposition follows from $[4$, Thm. 1].

Proof of the proposition. - (a) $\Rightarrow$ (b) is trivial; (b) $\Rightarrow$ (c) follows from the fact that $L(f, A, \mu)=\inf _{n} n^{-1} \int_{K} \log \left\|A_{n}\right\| \mathrm{d} \mu$.

We are left to prove (c) $\Rightarrow$ (a). Assume that (a) does not hold. Then there exists a sequence $x_{k} \in K, n_{k} \rightarrow \infty$ such that $\left\|A_{n_{k}}\left(x_{k}\right)\right\| \geq \mathrm{e}^{\varepsilon n_{k}}$. Let $\mu_{k}=n_{k}{ }^{-1} \sum_{j=0}^{n_{k}-1} \delta_{f^{j}\left(x_{k}\right)}$. Passing to a subsequence, we may assume that $\mu_{k}$ converges to $\mu$, which is $f$-invariant. We claim that $L(f, A, \mu) \geq \varepsilon$.

Let $\delta>0$ and $s \in \mathbb{N}$ be fixed. It is enough to show that

$$
\int \log \left\|A_{s}\right\| \mathrm{d} \mu \geq(\varepsilon-\delta) s
$$

Let $m_{k}=\left\lfloor n_{k} / s\right\rfloor$. Let $\nu_{k}=\left(s m_{k}\right)^{-1} \sum_{j=0}^{s m_{k}-1} \delta_{f^{j}\left(x_{k}\right)}$. Notice that $\nu_{k} \rightarrow \mu$. It is clear that if $k$ is large then $\left\|A_{s m_{k}}\left(f^{i}\left(x_{k}\right)\right)\right\| \geq \mathrm{e}^{(\varepsilon-\delta) s m_{k}}$ for $0 \leq i \leq s-1$. Then

$$
\begin{aligned}
\int \log \left\|A_{s}\right\| d \nu_{k} & =\frac{1}{s m_{k}} \sum_{i=0}^{s-1} \sum_{j=0}^{m_{k}-1} \log \left\|A_{s}\left(f^{j s+i}\left(x_{k}\right)\right)\right\| \\
& \geq \frac{1}{s m_{k}} \sum_{i=0}^{s-1} \log \left\|A_{s m_{k}}\left(f^{i}\left(x_{k}\right)\right)\right\| \geq s(\varepsilon-\delta) .
\end{aligned}
$$

The result follows.

\section{Perturbation along segments of orbits}

In this section we assume that $f: K \rightarrow K$ is minimal with no periodic orbits and $A: K \rightarrow \mathrm{SL}(2, \mathbb{R})$ is a continuous map such that $(f, A)$ is not uniformly hyperbolic, but there exists an $f$-invariant measure such that $L(f, A, \mu)>0$. The aim here is to establish Lemma 2 (see below).

We begin with an adaptation of Lemma 3.4 from [5]:

Lemma 1. - For every $\varepsilon>0$, there exists a non-empty open set $W \subset K$, and $m \in \mathbb{N}$ such that:

- $W, f(W), \ldots, f^{m-1}(W)$ are disjoint;

- for all $x \in W$ and any non-zero vectors $\boldsymbol{v}, \boldsymbol{w}$, there exists $M_{0}, \ldots, M_{m-1}$ in $\mathrm{SL}(2, \mathbb{R})$ such that $\left\|M_{j}-A\left(f^{j}(x)\right)\right\|<\varepsilon$ and $M_{m-1} \cdots M_{0}(\boldsymbol{v})$ is collinear to $\boldsymbol{w}$. 
Proof. - By assumption, there exists a $f$-invariant measure $\mu$ with nonvanishing exponents. We can assume $\mu$ is ergodic (and hence non-atomic). Then [5, Lemma 3.4] gives $m \in \mathbb{N}$ and a set $W$ which has all properties we want, except it is not necessarily open. (Notice that [5] gives the perturbed matrices in $\operatorname{GL}(2, \mathbb{R})$, but that is trivial to remedy.) Reducing $W$, we can assume it to be a point. Using the continuity of $A$, it is easy to see that $W$ can then be slightly enlarged to become open.

LEMMA 2. - Given $\varepsilon>0$, there exists arbitrarily large $N \in \mathbb{N}$ such that for every $x \in K$ there exist $L_{0}, \ldots, L_{N-1}$ in $\mathrm{SL}(2, \mathbb{R})$ satisfying

$$
\left\|L_{j}-A\left(f^{j}(x)\right)\right\|<\varepsilon \quad \text { and } \quad\left\|L_{N-1} \cdots L_{0}\right\|<\mathrm{e}^{\varepsilon N} .
$$

Lemma 2 is an adaptation of Lemma 5.1 from [1]. We will prove it using Lemma 1. If $A \in \mathrm{SL}(2, \mathbb{R}) \backslash \mathrm{SO}(2, \mathbb{R})$, then let $\boldsymbol{u}_{A}$ and $\boldsymbol{s}_{A}$ be unit vectors such that

$$
\left\|A\left(\boldsymbol{u}_{A}\right)\right\|=\|A\| \quad \text { and } \quad\left\|A\left(s_{A}\right)\right\|=\|A\|^{-1} .
$$

(Note that $\|$.$\| always denotes euclidian norm.) These vectors are unique mod-$ ulo multiplication by -1 . Notice that $\boldsymbol{u}_{A}$ is orthogonal to $\boldsymbol{s}_{A}$, and $A\left(\boldsymbol{u}_{A}\right)$ is collinear to $\boldsymbol{s}_{A^{-1}}$.

Proof of Lemma 2. - Let $W$ and $m$ be given by Lemma 1. Since $W$ is open and $f$ is minimal, there exists $m_{1} \in \mathbb{N}$ such that

$$
\bigcup_{j=0}^{m_{1}} f^{j}(W)=K
$$

Let $C>\varepsilon+\sup _{x}\|A(x)\|$. Take any $N \in \mathbb{N}$ be such that

$$
C^{4 m_{1}+1}<\frac{\mathrm{e}^{\varepsilon N}}{\sqrt{2}}
$$

From now on, let $x \in K$ be fixed. We will explain how to find matrices $L_{j}$ 's as in the statement of the lemma. Let

$$
\Delta_{j}=\frac{\left\|A_{j}(x)\right\|}{\left\|A_{N-j}\left(f^{j}(x)\right)\right\|}, \quad \text { for } 0 \leq j \leq N .
$$

Then $\Delta_{N}=\Delta_{0}^{-1}$ and $C^{-2}<\Delta_{j+1} / \Delta_{j}<C^{2}$. It follows that there exists $j_{0}$ such that $C^{-1}<\Delta_{j_{0}}<C$.

Due to (1), there exists $j_{1}$ such that $j_{0} \leq j_{1} \leq j_{0}+m_{1}$ and $f^{j_{1}}(x) \in W$. We can assume that $j_{1}+m \leq N$, because otherwise we would have $N<j_{0}+2 m_{1}$, so

$$
\left\|A_{N}(x)\right\|=\Delta_{N} \leq \Delta_{j_{0}} C^{4 m_{1}} \leq C^{4 m_{1}+1}<\mathrm{e}^{\varepsilon N},
$$

and then there would be nothing to prove. 
Let $X=A_{j_{1}}(x)$ and $Z=A_{N-j_{1}-m}\left(f^{j_{1}+m}(x)\right)$. Let $M_{0}, \ldots, M_{m-1}$ be given by Lemma 1 so that $\left\|M_{i}-A\left(f^{j_{1}+i}(x)\right)\right\|<\varepsilon$ and $M_{m-1} \cdots M_{0}\left(\boldsymbol{s}_{X^{-1}}\right)$ is collinear to $s_{Z}$. For $0 \leq j<N$, let

$$
L_{j}= \begin{cases}M_{j-j_{1}} & \text { if } j_{1} \leq j<j_{1}+m, \\ A\left(f^{j}(x)\right) & \text { otherwise }\end{cases}
$$

Write $Y=M_{m-1} \cdots M_{0}$, so $L_{N-1} \cdots L_{0}=Z Y X$. Notice $Y X \cdot \boldsymbol{u}_{X}$ is collinear to $s_{Z}$. So

$$
\begin{aligned}
\left\|Z Y X\left(\boldsymbol{u}_{X}\right)\right\| & \leq\left\|A_{j_{0}}(x)\left(\boldsymbol{u}_{X}\right)\right\| \cdot C^{j_{1}-j_{0}} \cdot\|Y\| \cdot\left\|Z\left(\boldsymbol{s}_{Z}\right)\right\| \\
& \leq\left\|A_{j_{0}}(x)\right\| \cdot C^{m_{1}+m} \cdot\|Z\|^{-1} \\
& \leq C^{2 m_{1}+2 m}\left\|A_{j_{0}}(x)\right\| \cdot\left\|A_{N-j_{0}}\left(f^{j_{0}}(x)\right)\right\|^{-1} \\
& =C^{2 m_{1}+2 m} \Delta_{j_{0}}<C^{4 m_{1}+1} .
\end{aligned}
$$

Also,

$$
\begin{aligned}
\left\|Z Y X\left(s_{X}\right)\right\| & \leq\|X\|^{-1} \cdot\|Y\| \cdot\|Z\| \\
& \leq\left\|A_{j_{0}}(x)\right\|^{-1} C^{m_{1}} \cdot C^{m} \cdot C^{m_{1}}\left\|A_{N-j_{0}}\left(f^{j_{0}}(x)\right)\right\| \\
& =C^{2 m_{1}+m} \Delta_{j_{0}}^{-1}<C^{3 m_{1}+1} .
\end{aligned}
$$

We have shown that $\max \left(\left\|Z Y X\left(\boldsymbol{u}_{X}\right)\right\|,\left\|Z Y X\left(\boldsymbol{s}_{X}\right)\right\|\right)<C^{4 m_{1}+1}<\mathrm{e}^{\varepsilon N} / \sqrt{2}$. Since $\boldsymbol{u}_{X} \perp \boldsymbol{s}_{X}$, we conclude that $\|Z Y X\|<\mathrm{e}^{\varepsilon N}$, as wanted.

\section{Tiling $K$}

A Borel set $X \subset K$ is said to be a zero probability set for the homeomorphism $f: K \rightarrow K$ if $\mu(X)=0$ for every $f$-invariant probability measure $\mu$.

Our goal in this section is to prove Lemma 3 below.

Lemma 3. - Let $f: K \rightarrow K$ be a homeomorphism of a compact set of finite dimension with no periodic orbits. There exists a basis of the topology of $K$ consisting of sets $U$ such that $\partial U$ is a zero probability set.

To prove Lemma 3, we will need Lemmas 4 and 5.

Lemma 4. - Let $f: K \rightarrow K$ be a homeomorphism of a compact set of finite dimension. Then there exists $d>0$, an embedding $s: K \rightarrow \mathbb{R}^{d}$ and $a$ homeomorphism $g: \mathbb{R}^{d} \rightarrow \mathbb{R}^{d}$ such that $s \circ f=g \circ s$.

TOME $135-2007-\mathrm{N}^{\mathrm{O}} 3$ 
Proof. - This result is well known, but we reproduce the proof for convenience. We may assume that $K \subset \mathbb{R}^{n}$ for some $n$. Let $\phi: \mathbb{R}^{n} \rightarrow \mathbb{R}^{n}$ and $\psi: \mathbb{R}^{n} \rightarrow \mathbb{R}^{n}$ be continuous extensions of $f$ and $f^{-1}$, respectively. Let $d=2 n, s(x)=(x, f(x))$ and $g(x, y)=(y, x+\phi(y)-\psi(y))$.

Lemma 5. - Let $f: \mathbb{R}^{d} \rightarrow \mathbb{R}^{d}$ be a homeomorphism and let $S$ be a compact manifold of dimension $d-1$. For a generic continuous $\psi: S \rightarrow \mathbb{R}^{d}$, and for every sequence of integers $j_{0}<\cdots<j_{d}, \bigcap_{k=0}^{d} f^{j_{k}}(\psi(S))$ is contained in the set of periodic orbits of $f$.

Proof. - Let $g_{i}: V_{i} \rightarrow \mathbb{R}^{d}$ be a countable family of charts so that there are exists a basis of the topology of $S$ formed by sets $U_{i}$ such that $\bar{U}_{i} \subset V_{i}$. Given sequences $\boldsymbol{j}=\left(j_{0}<\cdots<j_{d}\right)$ and $\boldsymbol{i}=\left(i_{0}, \ldots, i_{d}\right)$ such that $\bar{U}_{i_{0}}, \ldots, \bar{U}_{i_{d}}$ are disjoint, let $\mathcal{U}_{\boldsymbol{j}, \boldsymbol{i}}$ be the set of continuous maps $\psi: S \rightarrow \mathbb{R}^{d}$ such that

$$
\bigcap_{k=0}^{d} f^{j_{k}} \circ \psi\left(\bar{U}_{i_{k}}\right)=\varnothing .
$$

Claim 1. - The set $\mathcal{U}_{j, i}$ is open and dense in $C\left(S, \mathbb{R}^{d}\right)$.

Assuming the claim for the moment, let us conclude the proof of the lemma. Let $\psi$ belong to the residual set $\bigcap \mathcal{U}_{\boldsymbol{j}, \boldsymbol{i}}$. Assume that $z \in \bigcap_{k=0}^{d} f^{j_{k}}(\psi(S))$ for some $j_{0}<\cdots<j_{d}$. Let $x_{k} \in S$ be such that $f^{j_{k}}\left(\psi\left(x_{k}\right)\right)=z$, for $0 \leq k \leq d$. If all $x_{k}$ where distinct then we could take chart domains $U_{i_{k}} \ni x_{k}$ with $\bar{U}_{i_{0}}$, $\ldots, \bar{U}_{i_{d}}$ disjoint. This would contradict $\psi \in \mathcal{U}_{\boldsymbol{j}, \boldsymbol{i}}$. We conclude that at least two $x_{k}$ 's coincide. It follows that $z$ is a periodic point of $f$.

Now, the proof of the claim. Openness is clear; it remains to show denseness. For simplicity of writing, let $i_{k}=k$. Reducing the sets $V_{k}$ if necessary, we can assume they are disjoint (but still with $V_{k} \supset \bar{U}_{k}$ ). By a small perturbation of $\psi$ supported on $V_{k}$, we may assume that $f^{j_{k}} \circ \psi \circ g_{k}^{-1}$ is smooth in a neighborhood of $g_{k}\left(U_{k}\right)$. In other words, letting $L=\overline{g_{0}\left(U_{0}\right)} \times \cdots \times \overline{g_{d}\left(U_{d}\right)} \subset\left(\mathbb{R}^{d-1}\right)^{d+1}$, there is a neighborhood $W \supset L$ such that the map

$$
G: W \rightarrow\left(\mathbb{R}^{d}\right)^{d+1}, \quad G=\left(f^{j_{0}} \circ \psi \circ g_{0}^{-1}, \ldots, f^{j_{d}} \circ \psi \circ g_{d}^{-1}\right)
$$

is smooth. Perturbing $\psi$ again, we may assume that $G$ is transverse to the diagonal $D=\left\{(x, \ldots, x) \in\left(\mathbb{R}^{d}\right)^{d+1} ; x \in \mathbb{R}^{d}\right\}$ at $L$. Since the diagonal has codimension $d^{2}$ and $W$ has dimension $d^{2}-1$, this implies that $G(L)$ does not intersect $D$. That is, $\bigcap_{k=0}^{d} f^{j_{k}} \circ \psi\left(\bar{U}_{k}\right)=\varnothing$.

Proof of Lemma 3. - By Lemma 4, we can assume that $K \subset \mathbb{R}^{d}$ and $f: K \rightarrow K$ is the restriction of a homeomorphism $f: \mathbb{R}^{d} \rightarrow \mathbb{R}^{d}$.

Let $x_{0} \in K$, and let $\varepsilon>0$. We need to show that there exists an open set $U \subset \mathbb{R}^{d}$ containing $x_{0}$ and of diameter at most $4 \varepsilon$, such that $\mu(\partial U)=0$ for every $f$-invariant probability $\mu$ supported on $K$. 
Let $B$ be the closed unit ball in $\mathbb{R}^{d}$, and let $S=\partial B$. Let $\phi: B \rightarrow \mathbb{R}^{d}$ be given by $\phi(y)=x_{0}+\varepsilon y$. By Lemma 5 , there exists a continuous map $\psi: S \rightarrow \mathbb{R}^{d}$ such that $\|\psi(y)-\phi(y)\|<\varepsilon$ for every $y \in S$ and such that for every $x \in K$, the set of $\ell \in \mathbb{Z}$ such that $f^{-\ell}(x) \in \psi(S)$ has cardinality at most $d+1$. By the Ergodic Theorem, $\mu(\psi(S))=0$ for every $f$-invariant probability measure. Let $U$ be the connected component of $x_{0}$ in $\mathbb{R}^{d} \backslash \psi(S)$. Then the diameter of $U$ is at most $4 \varepsilon$ and $\partial U \subset \psi(S)$, so $U$ has all the desired properties.

\section{Proof of Theorem 1}

Let $f: K \rightarrow K$ be a minimal homeomorphism of a compact set of finite dimension. From now on, we assume that $f$ does not have periodic points; otherwise the result is obvious.

The idea of the following lemma and its proof come from [3].

Lemma 6. - For any $N \in \mathbb{N}$, there exists an open set $B \subset K$ such that:

- the return time from $B$ to itself via $f$ assumes the values $N$ and $N+1$ only;

- $\partial B$ has zero probability.

Proof. - Given $N$, there exists $n_{1} \in \mathbb{N}$ such that if $n \geq n_{1}$ then there are $\ell, \ell^{\prime} \geq 0$ satisfying $n=\ell N+\ell^{\prime}(N+1)$. By Lemma 3 and the fact that $f$ has no periodic points, we can take an open set $U$ such that $\partial U$ has zero probability and $U, f(U), \ldots, f^{n_{1}}(U)$ are disjoint. Then, for some $n_{2}>n_{1}$, $\bigcup_{n=0}^{n_{2}} f^{n}(U)=K$. For each $n \in \mathbb{N}$, let

$$
U_{n}=U \cap f^{-n}(U) \backslash \bigcup_{j=1}^{n-1} f^{-j}(U),
$$

that is, the set of points $x \in U$ such that the first return time to $U$ is $n$. Notice that $\partial U_{n}$ has zero probability. For each $n$ between $n_{1}$ and $n_{2}$ for which $U_{n} \neq \varnothing$, consider the tower of height $n$ with base $U_{n}$. Then break this tower into towers of heights $N$ or $N+1$. In this way we cover $K$ with finitely many towers of heights $N$ or $N+1$. Let $B$ be the union of the bases of the towers. Since $B$ is a finite union of iterates of $U_{n}$ 's, we have that $\partial B$ has zero probability.

LEMMA 7. - If $L$ is a compact set with zero probability then for every $\varepsilon>0$, there exists an open set $V \supset L$ and $n_{0} \in \mathbb{N}$ such that

$$
\frac{1}{n} \#\left\{j ; 0 \leq j \leq n-1, f^{j}(x) \in V\right\}<\varepsilon \quad \text { for all } x \in K, n \geq n_{0} .
$$

TOME $135-2007-\mathrm{N}^{\mathrm{O}} 3$ 
Proof. - Otherwise there exist $\varepsilon>0, x_{k} \in K$, open sets $V_{k} \subset K$ and integers $n_{k} \rightarrow \infty$ such that $\bar{V}_{k+1} \subset \operatorname{int} V_{k}, \bigcap V_{k}=L$ and $\mu_{k}\left(V_{k}\right)>\varepsilon$, where $\mu_{k}=n_{k}{ }^{-1} \sum_{j=0}^{n_{k}-1} \delta_{f^{j}\left(x_{k}\right)}$ (here $\delta_{x}$ is the Dirac mass on $x$ ). If $\mu$ is a weak-* limit of the probability measures $\mu_{k}$ then $\mu$ is $f$-invariant. Moreover, we have $\mu\left(V_{k}\right) \geq \liminf \mu_{j}\left(V_{j}\right) \geq \varepsilon$ for every $k$. We conclude that $\mu(L) \geq \varepsilon$, contradiction.

Proof of Theorem 1. - The set $U H$ of continuous $A: K \rightarrow \mathrm{SL}(2, \mathbb{R})$ such that $(f, A)$ is uniformly hyperbolic is open. For any $\varepsilon>0$, let $\mathcal{U}_{\varepsilon}$ be the set of $A$ 's such that there is $n_{1} \in \mathbb{N}$ such that $\frac{1}{n_{1}} \log \left\|A_{n_{1}}(x)\right\|<\varepsilon$ for all $x \in K$; then $\mathcal{U}_{\varepsilon}$ is also an open set. If $A \in \bigcap_{\varepsilon>0} \mathcal{U}_{\varepsilon}$ then $(f, A)$ has uniform subexponential growth, by Proposition 1. So to prove the theorem it suffices to show that $\mathcal{U}_{\varepsilon}$ is dense in the complement of $U H$.

Fix $\varepsilon>0$ and $A \notin U H$. We can assume that $(f, A)$ has positive exponent for some invariant probability, otherwise (by Proposition 1) $f \in \mathcal{U}_{\varepsilon}$ already. We will find $\widetilde{A}$ close to $A$ such that $\widetilde{A} \in \mathcal{U}_{\varepsilon}$.

Let $c>0$ be such that $\|A\|+\varepsilon<\mathrm{e}^{c}$. Let $N \in \mathbb{N}$ be given by Lemma 2 (notice hypotheses from $\S 3$ hold). We can assume $\varepsilon N>c$. Then let $B \subset K$ be the set given by Lemma 6 . Let $\delta>0$ be small enough so that

$$
0 \leq j \leq N, d(x, y)<\delta \Longrightarrow\left\|A\left(f^{j}(x)\right)-A\left(f^{j}(y)\right)\right\|<\varepsilon .
$$

Cover the closure of $B$ by open sets $W_{1}, \ldots, W_{k}$ with diameter less than $\delta$. By Lemma 3 , we can take each $W_{i}$ so that $\partial W_{i}$ has zero probability. Let $U_{i}=W_{i} \backslash \bigcup_{j<i} \bar{W}_{j}$.

Let $B_{\ell}$ be the set of points in $B$ whose first return to $B$ occurs in time $\ell$; then $B=B_{N} \sqcup B_{N+1}$. Let

$$
L=\bigcup_{\ell=N}^{N+1} \bigcup_{i=0}^{\ell-1} \partial\left(B_{\ell} \cap U_{i}\right) .
$$

Then $L$ has zero probability. By Lemma 7, there exists an open set $V \supset L$ and $n_{0} \in \mathbb{N}$ such that

(3) $\frac{1}{n} \#\left\{j ; 0 \leq j \leq n-1, f^{j}(x) \in V\right\}<\frac{\varepsilon}{N+1}$ for all $x \in K, n \geq n_{0}$.

For each $\ell=N$ or $N+1$ and $i=1, \ldots, k$, we choose a point $x_{\ell, i}$ in $B_{\ell} \cap U_{i}$. Applying Lemma 2, we find $L_{\ell, i, 0}, \ldots, L_{\ell, i, N-1}$ so that

$$
\left\|L_{\ell, i, j}-A\left(f^{j}\left(x_{\ell, i}\right)\right)\right\|<\varepsilon, \quad \forall j=0, \ldots, \ell-1
$$

and $\left\|L_{\ell, i, N-1} \cdots L_{\ell, i, 0}\right\|<\mathrm{e}^{\varepsilon N}$. In the case $\ell=N+1$, define also

$$
L_{\ell, i, N}=A\left(f^{j}\left(x_{\ell, i}\right)\right) .
$$

So for any $\ell,\left\|L_{\ell, i, \ell-1} \cdots L_{\ell, i, 0}\right\|<\mathrm{e}^{2 \varepsilon \ell}$. 
We want to define a map $\widetilde{A}: K \rightarrow \mathrm{SL}(2, \mathbb{R})$. Begin defining

$$
\widetilde{A}=L_{\ell, i, j} \text { on the set } f^{j}\left(B_{\ell} \cap U_{i} \backslash V\right),
$$

where $N \leq \ell \leq N+1,1 \leq i \leq k$, and $0 \leq j \leq \ell-1$. (Notice these sets are disjoint.) It remains to define $\widetilde{A}$ on the rest of $K$. The map $A^{-1} \widetilde{A}$ is already defined on a compact subset of $K$, and takes values on the $\left(\mathrm{e}^{c}+1\right) \varepsilon$-neighborhood of $\operatorname{Id}$ in $\operatorname{SL}(2, \mathbb{R})$. That domain is homeomorphic to $\mathbb{R}^{3}$ (provided $\varepsilon$ is not too big), so by the Tietze's extension theorem we can continuously extend $A^{-1} \widetilde{A}$ to the whole $K$. So we obtain $\widetilde{A}: K \rightarrow \mathrm{SL}(2, \mathbb{R})$ with $\|\widetilde{A}-A\|<\mathrm{e}^{c}\left(\mathrm{e}^{c}+1\right) \varepsilon$.

Now let $n>\max \left(n_{0},(N+1) / \varepsilon\right)$. Fix $x \in K$. We will give an upper bound for $\left\|\widetilde{A}_{n}(x)\right\|$. Write

$$
n=p+\ell_{1}+\ell_{2}+\cdots+\ell_{r}+q
$$

in a way such that $0 \leq p, q \leq N+1, N \leq \ell_{i} \leq N+1$, and the points

$$
x_{1}=f^{p}(x), x_{2}=f^{p+\ell_{1}}(x), \ldots, x_{r}=f^{p+\ell_{1}+\cdots+\ell_{r}}(x)
$$

are exactly the points in the segment of orbit $x, f(x), \ldots, f^{n-1}(x)$ that belong to $B$. We have

$$
\left\|A_{n}(x)\right\| \leq \mathrm{e}^{2 c(N+1)} \prod_{i=1}^{r}\left\|A_{\ell_{i}}\left(x_{i}\right)\right\| .
$$

We will use the bounds

$$
\left\|A_{\ell_{i}}\left(x_{i}\right)\right\| \leq \begin{cases}\mathrm{e}^{2 \varepsilon \ell_{i}} & \text { if } x_{i} \in B \backslash V, \\ \mathrm{e}^{c(N+1)} & \text { if } x_{i} \in V .\end{cases}
$$

By $(3)$, there are at most $(\varepsilon /(N+1)) n$ points $x_{i}$ that belong to $V$. So

$$
\left\|A_{n}(x)\right\| \leq \mathrm{e}^{2 c(N+1)} \cdot\left(\mathrm{e}^{c(N+1)}\right)^{(\varepsilon /(N+1)) n} \cdot \mathrm{e}^{2 \varepsilon \sum \ell_{i}}<\mathrm{e}^{(3 c+2) \varepsilon n} .
$$

This proves that $\widetilde{A} \in \mathcal{U}_{(3 c+2) \varepsilon}$.

\section{BIBLIOGRAPHY}

[1] J. BocHI - "Genericity of zero Lyapunov exponents", Ergodic Theory Dynam. Systems 22 (2002), p. 1667-1696.

[2] J. Bochi \& M. VianA - "The Lyapunov exponents of generic volumepreserving and symplectic maps", Ann. of Math. (2) 161 (2005), p. 14231485 .

[3] S. J. Eigen \& V. S. PRASAD - "Multiple Rokhlin tower theorem: a simple proof", New York J. Math. 3A (1997/98), p. 11-14.

[4] A. Furman - "On the multiplicative ergodic theorem for uniquely ergodic systems", Ann. Inst. H. Poincaré Probab. Statist. 33 (1997), p. 797-815. 
[5] N. ĐìnH CốNG - "A generic bounded linear cocycle has simple Lyapunov spectrum", Ergodic Theory Dynam. Systems 25 (2005), p. 1775-1797.

[6] J.-I. NAGATA - Modern dimension theory, Bibliotheca Mathematica, Vol. VI. Edited with the cooperation of the "Mathematisch Centrum" and the "Wiskundig Genootschap" at Amsterdam, Interscience Publishers John Wiley \& Sons, Inc., New York, 1965.

[7] J.-C. YocCOZ - "Some questions and remarks about SL $(2, \mathbf{R})$ cocycles", in Modern dynamical systems and applications, Cambridge Univ. Press, 2004, p. 447-458. 